\title{
Qualidade de Vida e Diabetes Mellitus: Autopercepção de Adolescentes de uma Cidade do Sul do Brasil
}

\author{
Marina Menezes, ${ }^{1}$ " (D), Leo Lynce Valle de $\operatorname{Lacerda}^{2}$ (D), Jaqueline Borella ${ }^{2}$ (D), \\ $\&$ Talya Pedron Alves ${ }^{2}$ (D) \\ ${ }^{1}$ Universidade Federal de Santa Catarina (UFSC), Florianópolis, SC, Brasil \\ ${ }^{2}$ Universidade do Vale do Itajaí (UNIVALI), Itajai, SC, Brasil
}

\begin{abstract}
RESUMO - Objetivou-se, neste estudo, avaliar a Qualidade de Vida em Relação à Saúde (QVRS) de adolescentes de 10 a 18 anos, com diagnóstico de diabetes mellitus tipo 1 (DM1), usuários dos serviços de duas unidades de atenção secundária em saúde municipal, no sul do Brasil. Participaram do estudo 20 adolescentes de ambos os sexos, com idades de 10 a 18 anos, sendo os dados coletados através do Questionário KIDSCREEN-52 versão brasileira. Os resultados indicaram que os adolescentes apresentaram ótima percepção de QVRS e que ocorreram poucas diferenças significativas em relação a sexo, classes socioeconômicas, idade e tempo de diagnóstico. Os resultados sugerem que a interferência da DM1 na QVRS deve ser analisada em função do processo de desenvolvimento da adolescência.
\end{abstract}

PALAVRAS-CHAVE: diabetes mellitus, qualidade de vida, adolescentes

\section{Quality of Life and Diabetes Mellitus: Perceptions of Teens on a Southern City of Brazil}

\begin{abstract}
This study aimed to evaluate the health-related quality of life (HRQOL) of adolescents aged 10 to 18 years, diagnosed with type 1 diabetes mellitus (T1DM), users of the services of two units of secondary care in municipal health in southern Brazil. The study included 20 adolescents of both sexes, aged 10-18 years, with the data collected through the questionnaire KIDSCREEN-52 Brazilian version. The results indicated teenagers who showed great perception of HRQOL and that there have been few significant differences concerning gender, socioeconomic status, age, and time of diagnosis. The results suggest that interference of T1DM in HRQOL should be analyzed in terms of adolescent development.
\end{abstract}

KEYWORDS: diabetes mellitus, quality of life, teenagers

A diabetes mellitus (DM), segundo dados da International Diabetes Federation (IDF, 2017) atingiu em 2017, 425 milhões de pessoas em todo o planeta e a perspectiva é de que esse número aumente para 629 milhões até 2045 (IDF, 2017; World Health Organization [WHO], 2016). O Brasil foi o quarto país do mundo com o maior número de pessoas com diabetes (12,4 milhões) e ocupa o terceiro lugar no ranking de países, por número de crianças menores de 20 anos com diabetes tipo 1 (DM1) (88,300 casos). A população mundial de crianças com DM1 foi de 1,106 milhões em 2017. Estima-se ainda que o número de novos casos de crianças diagnosticadas a cada ano seja de 100.000 (IDF, 2017).
De acordo com as Diretrizes da Sociedade Brasileira de Diabetes (Oliveira \& Vencio, 2016) a natureza crônica, a gravidade das complicações e os meios necessários para o controle da DM, a tornam onerosa para os indivíduos afetados, suas famílias e também para o sistema de saúde. Somente no ano de 2017, os gastos mundiais com o tratamento da diabetes e suas complicações alcançou 727 milhões de dólares (IDF, 2017).

Em termos nosográficos a DM configura-se como um grupo heterogêneo de distúrbios metabólicos que possui em comum a hiperglicemia. Etiologicamente resulta de defeitos na secreção ou na ação da insulina, ou em ambas

\footnotetext{
*E-mail: menezesmarina@yahoo.com.br

- Submetido: 20/01/2017; Aceito: 09/02/2018.
} 
(Oliveira \& Vencio, 2016). ADM1 (anteriormente conhecida como diabetes juvenil ou insulino-dependente, com início na infância) é caracterizada pela produção deficiente de insulina no corpo. A diabetes tipo 2 (anteriormente chamada de não-insulino-dependente ou diabetes do adulto) resulta da utilização ineficaz de insulina no corpo (WHO, 2016). Outros tipos específicos de diabetes resultam de defeitos genéticos na ação da insulina e doenças do pâncreas, entre outras condições e a diabetes gestacional está associada a qualquer intolerância à glicose, de magnitude variável, com início ou diagnóstico no período gestacional (Oliveira $\&$ Vencio, 2016).

Os estudos sobre a doença crônica são unânimes em considerar que a adolescência tende a ser o período mais difícil para este tipo de vivência. Assim, o início de uma doença que leve à necessidade de modificar muitos dos seus hábitos, gostos, estilos de vida, pode ser especialmente estressor. O fato de a adolescência ser uma fase de redescoberta do corpo, das suas potencialidades e limites, da sua capacidade de se definir como elemento de atração e relação com os outros, pode trazer toda a problemática da doença e do tratamento para o centro da atenção, levando o jovem a definir-se como particularmente diferente e "anormal" face ao grupo de pares, ou ao ideal de beleza juvenil (Barros, 2003).

Muitas crianças e adolescentes podem considerar difícil lidar emocionalmente com a doença, pois a vivência da DM1 pode resultar em discriminação, limitar as relações sociais e gerar impacto sobre o desempenho acadêmico. Os custos de tratamento e monitorização de equipamento, combinado com as necessidades diárias de uma criança com diabetes, podem representar um fardo financeiro e emocional significativo sobre toda a família. Assim, aos poucos, o adolescente deverá aprender a gerenciar sua vida com a DM, em um processo de busca pela qualidade de vida e autonomia (IDF, 2015).

O significado de qualidade de vida vai muito além da sintomatologia e dos efeitos da doença no estado funcional do indivíduo, abrangendo a importância dos sistemas de significação cultural, dos valores e preferências pessoais, para a qualidade de vida subjetiva (Paredes et al., 2008). As novas abordagens consideram a qualidade de vida como um construto que abarca o ajustamento psicossocial, bem-estar, autoestima, estresse e estratégias de enfrentamento (Matos et al., 2012).

Dessa forma, busca-se avaliar a percepção da criança e do adolescente sobre seu ambiente, suas preferências e a qualidade do seu mundo a partir das suas próprias perspectivas, incluindo a percepção de felicidade pessoal. O foco de tais avaliações não está centrado na presença ou ausência de déficits, mas nas qualidades positivas da criança, do adolescente e dos seus contextos, numa perspectiva ecológica, que considere variados níveis de análise, como a própria criança ou adolescente, sua família, pares, comunidade e escola (Matos et al., 2012).

Considerando este conjunto de aspectos relacionados, o presente estudo objetivou avaliar a qualidade de vida em relação à saúde para adolescentes diagnosticados com diabetes mellitus, bem como identificar as possíveis associações entre qualidade de vida, sexo, idade, classes socioeconômicas e tempo de diagnóstico.

\section{MÉTODO}

O presente estudo é descritivo, exploratório e de cunho quantitativo. Os locais da pesquisa foram duas Unidades de Atenção Secundária em Saúde municipal de uma cidade do sul do Brasil (Unidade A e Unidade B) ${ }^{1}$, cujo objetivo é atender aos principais agravos de saúde da população, com procedimentos e atendimentos especializados, quando não há necessidade internação.

\section{Participantes}

Os participantes foram adolescentes com DM1, contatados através da agenda de consultas do médico endocrinologista pediátrico que atua nestas unidades de saúde, e convidados a participar da pesquisa.

Os critérios de inclusão para a seleção dos participantes foram: a) idade entre 10 a 18 anos e 11 meses; b) diagnóstico de DM1; e c) ser usuário de uma das duas unidades (A ou B) de atenção secundária em saúde municipal. Considerou-se como critério de exclusão apresentar qualquer

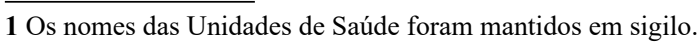

outra patologia, deficiência motora e/ou intelectual ou incapacidade para responder ao instrumento de coleta de dados da pesquisa.

A amostra deste estudo foi então composta por 20 adolescentes de ambos os sexos, entre 10 e 18 anos de idade, com diagnóstico de DM1. A Tabela 1 descreve outras características dos participantes da pesquisa em relação ao tempo de diagnóstico de DM1 e classe socioeconômica ${ }^{2}$.

\section{Instrumento}

Os dados referentes à qualidade de vida foram coletados através do Questionário de Qualidade de vida

2 Para fins de padronização, utilizou-se o modelo de dados calculados com base no Critério de Classificação Econômica Brasil da Associação Brasileira de Empresas de Pesquisa (ABEP, 2016), sendo a renda média domiciliar equiparada aos seguintes valores: $(\mathbf{A}=\mathrm{R} \$ 20.888,00 ; \mathbf{B} 1=$ $\mathrm{R} \$ 9.254,00 ; \mathbf{B 2}=\mathrm{R} \$ 4.852,00 ; \mathbf{C} 1=\mathrm{R} \$ 2.705,00 ; \mathbf{C} 2=\mathrm{R} \$ 1.625,00 ;$ $\mathbf{D}-\mathbf{E}=\mathrm{R} \$ 768,00)$. Valor do salário mínimo no Brasil, calculado em reais, segundo o Ministério do Trabalho e Previdência Social: R \$ 880,00 (valor do salário mínimo de 2016, de acordo com o Ministério do Trabalho. Fonte: http://trabalho.gov.br/salario-minimo ). 
Tabela 1

Caracterização da amostra de participantes da pesquisa

\begin{tabular}{|c|c|c|}
\hline Característica & Número & Percentual \\
\hline \multicolumn{3}{|l|}{ Sexo } \\
\hline Masculino & 7 & 35,0 \\
\hline Feminino & 13 & 65,0 \\
\hline \multicolumn{3}{|l|}{ Idade em anos } \\
\hline $10-14$ & 9 & 45,0 \\
\hline $15-18$ & 11 & 55,0 \\
\hline \multicolumn{3}{|c|}{ Tempo de Diagnóstico de DM1 em anos } \\
\hline $0-5$ & 8 & 40,0 \\
\hline $6-10$ & 12 & 60,0 \\
\hline \multicolumn{3}{|c|}{ Classe Socioeconômica } \\
\hline A & - & - \\
\hline B1 & 4 & 20,0 \\
\hline B2 & 7 & 35,0 \\
\hline $\mathrm{C} 1$ & 6 & 30,0 \\
\hline $\mathrm{C} 2$ & 3 & 15,0 \\
\hline $\mathrm{D}-\mathrm{E}$ & - & - \\
\hline
\end{tabular}

KIDSCREEN-52 versão brasileira, traduzido por Guedes e Guedes (2011). O KIDSCREEN-52 é um instrumento autoaplicável que pode ser utilizado para avaliar a saúde associada à qualidade de vida para crianças e adolescentes com idades entre 10 a 18 anos. É composto por dez dimensões que descrevem a qualidade de vida relacionada à: Saúde e Atividade Física (cinco itens); Sentimentos (seis itens); Estado Emocional (sete itens); Autopercepção (cinco itens); Autonomia e Tempo livre (cinco itens); Família e Ambiente Familiar (seis itens); Questões Econômicas (três itens); Amigos e Apoio Social (seis itens); Ambiente Escolar e Aprendizagem (seis itens) e Provocação - Bullying (três itens).

\section{Procedimento}

O presente estudo respeitou as diretrizes da Resolução $\mathrm{N}^{\circ} 466 / 2012$ do Conselho Nacional da Saúde (CNS) e foi aprovado pelo Comitê de Ética em Pesquisa da Universidade do Vale do Itajaí (UNIVALI), sob o parecer $\mathrm{N}^{\circ} 1.227 .049$ CAAE 45237715.2.0000.0120, de 14 de setembro de 2015.

Durante o período de coleta de dados, ocorrido entre agosto a outubro de 2015, o número de adolescentes com DM1 que recebiam assistência na Unidade A era 18, sendo que somente 6 puderam ser contatados devido à falta de atualização cadastral ${ }^{3}$. Dos adolescentes contatados, apenas 3 aceitaram participar da pesquisa. Já na Unidade B, 40 adolescentes recebiam assistência, sendo que foi

3 A atualização cadastral se refere à atualização das informações dos usuários, fornecidas na admissão dos serviços das unidades de saúde, tais como número de telefone, endereço residencial, etc. possível contatar apenas 28 usuários pelo mesmo motivo de desatualização cadastral. Dentre os contatados, 17 aceitaram participar da pesquisa. A coleta de dados foi realizada na residência dos adolescentes, com exceção de um participante que aceitou realizar a aplicação do instrumento em uma das salas disponíveis na Unidade B.

Os dados foram analisados através de estatística descritiva e inferencial. A caracterização da amostra foi realizada por meio de frequência simples e os escores do questionário KIDSCREEN-52 foram expressos pela mediana e desvio interquartílico de cada dimensão a ser avaliada. Nas inferências, as associações entre as dimensões e as variáveis socioeconômicas foram analisadas de acordo com a natureza das últimas. Para verificar as diferenças entre os sexos foi utilizada a prova não paramétrica de postos de Mann - Whitney (Siegel \& Castellan Junior, 2005), na qual os escores foram classificados crescentemente em postos, do mais baixo ao mais alto.

Para analisar as diferenças entre as dimensões e as classes socioeconômicas foi utilizada a prova não paramétrica de Kruskal - Wallis com a mesma classificação de postos. A relação entre a idade, tempo de diagnóstico e as dimensões foi analisada por meio do coeficiente de correlação de postos de Spearman.

Optou-se pelas provas não paramétricas para a análise dos dados considerando a não normalidade dos dados, o baixo número amostral e a natureza ordinal das variáveis coletadas. O nível de significância adotado foi de $10 \%$ em todas as análises, tendo em vista a variabilidade da percepção dos indivíduos. O aplicativo utilizado para as inferências foi o Statistica versão 10 (Statsoft, 2011). 


\section{RESULTADOS}

Pode-se observar que os maiores percentuais em relação ao sexo são do sexo feminino, representando $65 \%$ da amostra. No que se refere à idade em anos, 55\% dos adolescentes estavam na faixa entre 15 e 18 anos e $60 \%$ tinham seis ou mais anos de tempo de diagnóstico. Ao observar a classificação socioeconômica dos participantes, o maior percentil se refere à classe B2, significando $35 \%$ da amostra. É importante notar que não houve participantes nos extremos das classes A e D-E. De acordo com a Associação Brasileira de Empresas de Pesquisa (ABEP, 2016), na região sul do Brasil a maior concentração de pessoas está na classe C1, representando $28 \%$ da população e os menores índices, assim como constatado no presente estudo, são relativos às classes D-E $(17,0 \%)$ e A $(3,4 \%)$.

O escore médio da percepção de QVRS de modo geral dos adolescentes foi elevado $(83,7+/-12,8)$, com pontos medianos das dimensões acima de 60 , conforme demonstra a Tabela 2.

Os resultados indicam que os adolescentes da amostra apresentaram percepção da QVRS com escore 90 ou mais nas dimensões: Provocação - Bullying, Autonomia e Tempo Livre, Família e Ambiente Familiar, e menor que 70 na dimensão Saúde e Atividade Física, tendo sido a única dimensão com escore individual máximo abaixo de 100 . Nesta dimensão também se observou a maior variação individual de resposta, conforme pode ser visto por meio do maior desvio interquartílico $(28,0)$. Em contraposição, a dimensão Autopercepção mostrou homogeneidade de respostas, obtendo o menor desvio $(8,0)$.

A Tabela 3 mostra a análise da diferença dos escores de acordo com o sexo para cada uma das dimensões de qualidade de vida, não tendo sido identificadas diferenças significativas

Tabela 2

Distribuição das pontuações mínimas e máximas, medianas e desvios interquartílicos (desvio) nas dimensões do KIDSCREEN-52

\begin{tabular}{|c|c|c|c|c|}
\hline Dimensão & Mediana & Mínima & Máxima & Desvio \\
\hline Saúde e Atividade Física & 62,0 & 44,0 & 96,0 & 28,0 \\
\hline Sentimentos & 80,0 & 23,3 & 100,0 & 13,3 \\
\hline Estado Emocional & 78,6 & 31,4 & 100,0 & 22,9 \\
\hline Autopercepção & 84,0 & 24,0 & 100,0 & 8,0 \\
\hline Autonomia e Tempo Livre & 90,0 & 40,0 & 100,0 & 22,0 \\
\hline Família e Ambiente Familiar & 90,0 & 36,7 & 100,0 & 13,3 \\
\hline Questões Econômicas & 80,0 & 53,3 & 100,0 & 26,7 \\
\hline Amigos e Apoio Social & 76,7 & 36,7 & 100,0 & 21,7 \\
\hline Ambiente Escolar e Aprendizagem & 85,0 & 33,3 & 100,0 & 25,0 \\
\hline Provocação-Bullying & 100,0 & 60,0 & 100,0 & 16,7 \\
\hline Geral & 83,7 & 42,5 & 100,0 & 12,8 \\
\hline
\end{tabular}

Nota. $(\mathrm{n}=20)$.

Tabela 3

Escores medianos e significância de acordo com o sexo e a faixa etária nas dimensões do KIDSCREEN- 52

\begin{tabular}{|c|c|c|c|c|c|c|}
\hline \multirow[t]{2}{*}{ Dimensão } & \multicolumn{2}{|c|}{ Sexo } & \multicolumn{4}{|c|}{ Faixa Etária } \\
\hline & Feminino & Masculino & Valor $\mathrm{p}^{*}$ & $10-14$ & $15-18$ & Valor $\mathrm{p}^{*}$ \\
\hline Saúde e Atividade Física & 80,0 & 80,0 & 0,9368 & 72,0 & 56,0 & 0,4033 \\
\hline Sentimentos & 74,3 & 88,6 & 0,4281 & 83,3 & 76,7 & 0,0946 \\
\hline Estado Emocional & 80,0 & 88,0 & 0,0813 & 85,7 & 71,4 & 0,1837 \\
\hline Autopercepção & 92,0 & 88,0 & 0,1130 & 88,0 & 84,0 & 0,7612 \\
\hline Autonomia e Tempo Livre & 90,0 & 90,0 & 0,8430 & 88,0 & 92,0 & 1,0000 \\
\hline Família e Ambiente Familiar & 80,0 & 80,0 & 0,8741 & 86,7 & 90,0 & 0,7903 \\
\hline Questões Econômicas & 76,7 & 83,3 & 0,6065 & 80,0 & 80,0 & 0,7612 \\
\hline Amigos e Apoio Social & 86,7 & 83,3 & 0,7214 & 83,3 & 73,3 & 0,2545 \\
\hline Ambiente Escolar e Aprendizagem & 100,0 & 100,0 & 0,8121 & 90,0 & 73,3 & 0,0205 \\
\hline Provocação-Bullying & 80,0 & 80,0 & 0,6919 & 100,0 & 100,0 & 1,0000 \\
\hline
\end{tabular}

*De acordo com a Prova de Mann-Whitney ao nível de $10 \%$.

Nota. Optou-se pela apresentação da mediana ao invés da média dos postos tendo em vista que a mediana preserva a escala original de zero a 100 do escore de qualidade de vida.

${ }^{a}$ significância (valor de p). 
em todas, exceto na dimensão Estado Emocional, na qual o sexo feminino apresentou mediana significativamente menor do que o sexo masculino. A dimensão Autopercepção se aproximou do valor de significância $(p=0,1130)$, com mediana superior para o sexo feminino $(92,0)$ em relação ao masculino $(88,0)$.

Com relação à faixa etária, as dimensões Sentimentos e Ambiente Escolar e Aprendizagem apresentaram diferenças de escores significativas, com medianas maiores para a faixa etária de 10 a 14 anos (83,3 e 90 respectivamente) em contraste à faixa de 15 a 18 anos $(76,7$ e 73,3$)$.

Os resultados da análise das dimensões de acordo com as classes socioeconômicas são apresentados na Tabela 4.
Conforme pode ser observado, ocorreu diferença estatisticamente significativa $(p=0,0630)$ para as classes socioeconômicas, na dimensão Emocional. A percepção dos participantes da classe B2 (renda média mensal bruta familiar de R\$ 4.852,00) foi significativamente menor do que aquela da classe B1.

A análise das relações entre as dimensões de qualidade de vida com idade e o tempo de diagnóstico, apresentados na Tabela 5, mostrou correlações negativas e significativas de $41,5 \%$ na dimensão Sentimentos ( $p=0,0689$ ) e de $47,8 \%$ e na dimensão Escola $(p=0,0330)$ para a idade. Quando analisados os resultados para o tempo de diagnóstico, observou-se correlação positiva e significativa de $66,9 \%$ na dimensão Autonomia $(\mathrm{p}=0,0013)$.

Tabela 4

Escores medianos e significância de acordo com as dimensões do KIDSCREEN- 52 e classe socioeconômica

\begin{tabular}{|c|c|c|c|c|c|}
\hline \multirow[t]{2}{*}{ Dimensão } & \multicolumn{5}{|c|}{ Classe Socioeconômica } \\
\hline & $\begin{array}{c}\mathrm{B} 1 \\
(\mathrm{n}=4)\end{array}$ & $\begin{array}{c}\mathrm{B} 2 \\
(\mathrm{n}=3)\end{array}$ & $\begin{array}{c}\mathrm{C} 1 \\
(\mathrm{n}=6)\end{array}$ & $\begin{array}{c}\mathrm{C} 2 \\
(\mathrm{n}=3)\end{array}$ & Valor de $\mathrm{p}^{*}$ \\
\hline Saúde e Atividade Física & 72,0 & 64,0 & 58,0 & 76,0 & 0,9413 \\
\hline Sentimentos & 88,3 & 76,7 & 81,7 & 76,7 & 0,1894 \\
\hline Estado Emocional & 94,3 & 68,6 & 82,9 & 77,1 & 0,0630 \\
\hline Autopercepção & 92,0 & 80,0 & 81,0 & 84,0 & 0,1504 \\
\hline Autonomia e Tempo Livre & 92,0 & 84,0 & 96,0 & 88,0 & 0,8276 \\
\hline Família e Ambiente Familiar & 91,7 & 90,0 & 86,7 & 96,7 & 0,7236 \\
\hline Questões Econômicas & 96,7 & 80,0 & 76,7 & 73,3 & 0,2234 \\
\hline Amigos e Apoio Social & 90,0 & 73,3 & 81,7 & 63,3 & 0,1950 \\
\hline Ambiente Escolar e Aprendizagem & 93,3 & 83,3 & 86,7 & 80,0 & 0,6075 \\
\hline Provocação -Bullying & 100,0 & 86,7 & 100,0 & 100,0 & 0,1154 \\
\hline
\end{tabular}

*De acordo com a prova de Kruskal-Wallis. As letras sobrescritas iguais nas medianas denotam igualdade na prova de Dunn para os postos a posteriori. Nota. Optou-se pela apresentação da mediana ao invés da média dos postos tendo em vista que a mediana preserva a escala original de zero a 100 do escore de qualidade de vida.

a (número amostral em parênteses).

Tabela 5

Correlações e significância da relação entre as dimensões do KIDSCREEN-52, idade e tempo de diagnóstico dos participantes

\begin{tabular}{lcccc}
\hline Dimensão & Correlação Idade & Valor de p* & $\begin{array}{c}\text { Correlação } \\
\text { Diagnóstico }\end{array}$ & Valor de p* \\
\hline Saúde e Atividade Física & $-0,172$ & 0,4693 & 0,135 & 0,5691 \\
Sentimentos & $-0,415$ & 0,0689 & 0,288 & 0,2188 \\
Estado Emocional & $-0,181$ & 0,4453 & 0,192 & 0,4181 \\
Autopercepção & $-0,071$ & 0,7655 & 0,144 & 0,5445 \\
Autonomia e Tempo Livre & 0,110 & 0,6440 & 0,669 & 0,0013 \\
Família e Ambiente Familiar & 0,061 & 0,7973 & 0,187 & 0,4293 \\
Questões Econômicas & $-0,053$ & 0,8252 & 0,243 & 0,3026 \\
Amigos e Apoio Social & $-0,242$ & 0,3038 & 0,341 & 0,1414 \\
Ambiente Escolar e Aprendizagem & $-0,478$ & 0,0330 & 0,321 & 0,1680 \\
Provocação -Bullying & $-0,004$ & 0,9867 & 0,278 & 0,2355 \\
\hline
\end{tabular}

* Prova da correlação de postos de Spearman ao nível de $10 \%$.

Nota. Idade (em anos) e tempo de diagnóstico em anos.

${ }^{\mathrm{a}}(\mathrm{n}=20)$. 


\section{DISCUSSÃO}

Entre as hipóteses explicativas para o escore médio geral da percepção de QVRS dos adolescentes se apresentar elevado, mesmo considerando a DM1, destaca-se o fato de que os participantes deste estudo não se encontravam hospitalizados e todos estavam em acompanhamento ambulatorial para o tratamento da diabetes, ou seja, faziam uso de medidas de tratamento e controle da doença, como insulinoterapia, dietas, exercícios, exames de sangue frequentes e outros.

$\mathrm{Na}$ relação entre qualidade de vida e diabetes, o controle da glicemia representa uma medida importante por duas razões principais. Em primeiro lugar, para evitar a probabilidade de ocorrência de uma crise aguda (cetoacidose) que reduz a qualidade de vida, ocasionando internações hospitalares e aumentando a probabilidade de morte. Em segundo lugar, para evitar o desenvolvimento precoce de complicações crônicas, tais como danos aos rins e olhos, que também reduzem a qualidade de vida (Eiser \& Morse, 2001). Além destes aspectos, a insulinoterapia, as contribuições de familiares e o apoio emocional têm sido associados como fatores que melhoram a qualidade do controle metabólico de adolescentes com DM1 (Kakleas, Kandyla, Karayianni, \& Karavanaki, 2009).

A adoção e manutenção das medidas preconizadas no tratamento da diabetes parecem ter possibilitado uma percepção de QVRS mais positiva, conferindo algum controle sobre o tratamento e estabilidade com relação à saúde. Outro aspecto importante refere-se ao fato de que a QVRS é influenciada por diversos fatores pessoais, tais como autoestima e otimismo, e fatores sociais como as relações com a família e grupo de pares (Gaspar, Matos, Ribeiro, Leal, \& Ferreira, 2009).

A dimensão com escore médio mais elevado da amostra foi Provocação - Bullying, que se refere à percepção de aspectos relacionados aos sentimentos de rejeição pelos pares na escola. A indicação de valores elevados nesta dimensão ocorre quando os sentimentos negativos percebidos são raros (Gaspar \& Matos, 2008). Portanto, o escore médio dos participantes nesta dimensão revela que os mesmos não se sentem provocados ou rejeitados pelo grupo de pares, mas se sentem aceitos e respeitados. Resultados semelhantes foram observados no estudo de Perez (2013) ao investigar a qualidade de vida e as correlações com suporte social de 102 adolescentes de Porto Alegre, RS, com idades entre 12 e 17 anos e diagnóstico de DM1.

Muitas crianças e adolescentes com uma condição médica crônica apresentam mais vulnerabilidade de se tornarem vítimas de bullying em virtude de exibirem comportamentos de adesão ao tratamento ou apresentar diferenças físicas observáveis por seus pares (Peters, Storch, Geffken, Heidgerken, \& Silverstein, 2008).

Curiosamente, o fato de ter DM1, cujo controle e tratamento impõem uma série de restrições e representam condições que podem expor o adolescente ao estigma de sentir-se pressionado pelos pares em alguns contextos sociais, tais aspectos não afetaram a percepção do sentimento de aceitação e respeito dos colegas de grupo. Neste sentido, quanto maior a satisfação com a vida e a sensação de que a escola é um local seguro, menor é a frequência dos comportamentos de bullying entre os adolescentes (Matos \& Gonçalves, 2009; Salamon, Hains, Fleischman, Davies, $\&$ Kichler, 2010).

Contudo, pesquisas com o KIDSCREEN-52 em países europeus e na América Latina, revelaram que a percepção da dimensão Provocação - Bullying para crianças e adolescentes com sobrepeso, peso normal e sem doenças crônicas apresentou escores médios com a menor pontuação entre todas as dimensões avaliadas, indicando a percepção de sentimentos negativos intensos entre crianças e adolescentes com condições físicas e de saúde variadas (Guedes, Astudillo, Morales, Vecino, \& Pires Junior, 2014; Ottova, Erhart, Rajmil, Dettenborn-Betz, \& Ravens-Sieberer, 2012).

As dimensões Autonomia e Tempo Livre, e Família e Ambiente Familiar, também apresentaram escores médios elevados no presente estudo. A dimensão relativa à Autonomia e Tempo Livre se refere à oportunidade oferecida à criança ou adolescente de gerir seu tempo social e de lazer. Uma pontuação elevada revela a percepção de sentir-se livre para tomar decisões no cotidiano, ser independente e autônomo (Gaspar \& Matos, 2008).

O desenvolvimento da identidade também está relacionado à autonomia, assim, a experiência da transição de uma postura de receber cuidados de terceiros para uma em que é necessário desenvolver o autocuidado no tratamento e controle da diabetes, parece favorecer o adolescente a sentir-se mais capaz para gerenciar seu cotidiano de forma autossuficiente, incluindo a gerência das oportunidades para participar de atividades sociais e de lazer (Karlsson, Warman, \& Wickblad, 2008; Perez, 2013). Nesse sentido, a percepção da autonomia na avaliação da QVRS dos participantes mostrou-se relacionada à adoção de cuidados com o tratamento da DM1 que são assumidos e geridos pelos adolescentes em seu dia-a-dia.

A dimensão Família e Ambiente Familiar avalia a relação do adolescente com os pais e o ambiente vivenciado em casa. A pontuação elevada nesta dimensão indica a sensação de segurança, de sentir-se apoiado, amado, compreendido, bem cuidado e de perceber os pais como pessoas disponíveis e justas (Matos \& Gonçalves, 2009).

A DM1 é uma doença crônica com implicações na vida diária de adolescentes e suas famílias. Dado que a gestão da diabetes deve ser integrada ao estilo de vida familiar, é possível que a mesma afete cada membro particularmente. $\mathrm{O}$ aumento da responsabilidade e as demandas de tratamento pode levar a conflitos entre pais e filhos, pois o envolvimento 
parental pode se chocar com o desenvolvimento da autonomia do adolescente (Kakleas et al., 2009).

O apoio emocional ou instrumental parental nos cuidados com a diabetes relaciona-se com melhores resultados no controle da doença em adolescentes. Além disso, jovens que apresentam interações familiares mais positivas possuem um ambiente familiar mais coeso e pais mais colaborativos (Mackey et al., 2011). Desse modo, especula-se que os resultados referentes à dimensão Família e Ambiente Familiar deste estudo indiquem que os adolescentes apresentam a percepção de que são apoiados pela família e tratados com justiça pelos seus familiares, bem como percebem seu ambiente familiar como confortável.

$\mathrm{O}$ escore médio mais baixo entre todas as dimensões, na percepção dos participantes, foi observado na dimensão Saúde e Atividade Física, que se refere ao nível de atividade, energia e aptidão física do adolescente. A baixa pontuação nesta dimensão relaciona-se à sensação de exaustão física e baixa energia, e a não se sentir bem e em forma fisicamente (Gaspar \& Matos, 2008).

Outros estudos utilizando o KIDSCREEN-52 e 27 apresentaram resultados semelhantes. Perez (2013) também observou que a dimensão Saúde e Atividade Física obteve o escore médio mais baixo em um grupo de adolescentes brasileiros com DM1. Ottova et al. (2012) encontraram resultados similares com adolescentes europeus com sobrepeso, assim como González et al. (2014) em estudo com adolescentes colombianos com sobrepeso e obesidade.

O tratamento da DM1 envolve restrições alimentares, prática de exercícios físicos regulares, monitorização frequente do açúcar no sangue e a administração diária de insulina. Na perspectiva dos adolescentes da presente pesquisa, os sintomas da DM1 e o seu tratamento se associam à sensação de cansaço, baixa energia física e à percepção de não estar bem e em forma fisicamente.

Com relação ao sexo, observou-se uma diferença significativa apenas na dimensão Estado Emocional para as meninas e na dimensão Autopercepção para os meninos. De modo contrário, Perez (2013) identificou diferenças significativas entre os sexos em sua amostra de adolescentes com DM1, indicando que as médias das meninas foram mais baixas em quase todas as dimensões de qualidade de vida. Estudos realizados com adolescentes com e sem doenças crônicas também identificaram que os meninos apresentaram maiores médias nas dimensões de QVRS quando comparados às meninas (Gaspar \& Matos, 2008; González et al., 2014; Michel, Bisegger, Fuhr, Abel, \& KIDSCREEN Group, 2009).

A dimensão Estado Emocional, identificada como a dimensão pior avaliada pelas meninas do presente estudo, mede a vivência e a percepção de sentimentos e emoções depressivas e estressoras, tais como solidão, tristeza, suficiência/insuficiência e resignação. Escores baixos nesta dimensão indicam que o adolescente se percebe deprimido, infeliz e mal-humorado (Gaspar \& Matos, 2008).
Patton e Viner (2007) destacam a ênfase atribuída às consequências da puberdade em relação ao sexo, tais como as reações psicológicas mais negativas para mudanças corporais ou transições mais difíceis em papéis sociais e sexuais em meninas do que em meninos. Esta diferença por sexo é consistentemente observada em pesquisas que indicam que as adolescentes com DM1 têm resultados mais negativos relacionados à depressão, comportamentos de risco e puberdade precoce do que rapazes (Naar-King et al., 2006). Michel et al. (2009) complementam ressaltando que a partir dos 12 anos, adolescentes do sexo feminino apresentam percepção de QVRS com médias mais baixas do que os meninos em decorrência da menarca e do desequilíbrio hormonal.

Já a dimensão Autopercepção, pior avaliada pelos meninos da amostra, explora a percepção positiva ou negativa que o adolescente tem de si próprio, como a aparência do seu corpo, a satisfação com a aparência das roupas e outros acessórios pessoais. Uma pontuação baixa implica na percepção de uma imagem corporal negativa, infelicidade ou insatisfação consigo próprio, baixa autoestima e desconforto com a própria aparência (Gaspar $\&$ Matos, 2008).

Fatores como as mudanças físicas acarretadas pela adolescência, o sexo e o estado nutricional podem influenciar o adolescente na avaliação da sua imagem corporal e representam aspectos associados negativamente à mesma. Há também algumas evidências de que fatores socioculturais como a mídia, os colegas e os pais podem influenciar o desenvolvimento de uma imagem corporal negativa (Smolak, 2004).

A relação entre DM1 e o desenvolvimento físico de crianças e adolescentes tem apontado diferenças entre sexos, associando com maior frequência a obesidade e a baixa estatura no sexo feminino do que no sexo masculino (Elamin, Houssein, \& Tuvemo, 2006). No entanto, Smolak (2004) sinaliza que na adolescência, os meninos são mais propensos do que as meninas a querer aumentar o tamanho de seus músculos. Assim, é possível relacionar que os adolescentes do sexo masculino tenham sido influenciados por este conjunto de fatores na avaliação desta dimensão, percebendo-se de modo menos positivo do que as meninas, talvez movidos pelo ideal de aparentar um corpo maior e musculoso que talvez não seja atingido em decorrência da diabetes e das imposições do tratamento.

Com relação à idade, as dimensões Sentimentos e Ambiente Escolar e Aprendizagem foram avaliadas de forma mais positiva pelos adolescentes na faixa etária de 10 a 14 anos, em relação à faixa de 15 a 18 anos, caracterizando a ocorrência de decréscimo na avaliação destas dimensões com o avanço da idade. A dimensão Sentimentos avalia o bem-estar psicológico do adolescente, as emoções positivas e a satisfação com a vida. Já a dimensão Ambiente Escolar e Aprendizagem avalia a percepção que o adolescente tem da sua capacidade cognitiva, de aprendizagem e concentração, 
bem como a avaliação dos sentimentos em relação à escola enquanto ambiente agradável e a relação com os professores (Gaspar \& Matos, 2008).

Os resultados de pesquisas com o KIDSCREEN também indicaram decréscimo das médias de QVRS em geral com relação ao aumento da idade, tanto para meninos, quanto para meninas (Gaspar \& Matos, 2008; Michel et al., 2009; Perez, 2013). Alguns aspectos relacionados à menor pontuação atribuída às dimensões da QVRS estão associados ao avanço da idade na adolescência, sendo justificados por aspectos comportamentais (Guedes et al., 2014). Ao crescer, o adolescente confronta-se com uma transição física e social, sendo necessária uma adaptação corporal, fisiológica e de identidade de gênero (Patton \& Viner, 2007). No caso de adolescentes com DM1, a presença de eventos estressores como os procedimentos médicos e as internações hospitalares, bem como as exigências diárias de autocuidado, através do controle da dieta e da aplicação de insulina, além das limitações de atividades cotidianas representam fatores que podem acarretar na percepção de menores índices de QVRS ao longo dos anos (SeiffgeKrenke \& Stemmler, 2003).

Com relação ao ambiente escolar, as formas como as fontes de apoio social são percebidas variam de acordo com a idade e a etapa do desenvolvimento. Bockhorst, Sumter e Westenberg (2010) avaliaram as diferenças de idade e sexo na percepção do apoio social recebido dos pais, amigos, colegas de classe e professores entre crianças e adolescentes holandeses, com idades de 9 a 18 anos, sem doenças crônicas. Os resultados indicaram menor percepção de apoio dos professores para os adolescentes de 13 a 18 anos do ensino secundário. Neste período escolar os adolescentes se relacionam com vários professores e não desenvolvem relações pessoais com todos eles. Nas idades entre 9 a 12 anos, o número de professores é menor e as relações com eles são mais próximas. Os resultados deste estudo corroboram os achados referentes à diminuição do contato entre aluno e professor ocorrendo significativamente com o avanço da idade e afetando a percepção do apoio recebido.

Quanto aos aspectos socioeconômicos, observou-se diferença apenas na avaliação da dimensão Emocional na percepção dos participantes da classe B2 (renda média mensal bruta familiar de R $\$ 4.852,00$ ), significativamente menor do que da classe B1 (R $\$ 9.254,00$ ). Nesse sentido, infere-se que os adolescentes da classe B2 mostraram-se mais suscetíveis à percepção de sentimentos relativos a depressão, infelicidade e mau humor, do que adolescentes com maior poder aquisitivo. Segundo Bradley e Corwyn (2002) as crianças de famílias com níveis socioeconômicos inferiores são mais propensas a ter níveis mais baixos de bem-estar emocional e mais problemas comportamentais.
Gonzáles et al. (2014) avaliaram QVRS, aspectos sociodemográficos, excesso de peso, obesidade e atividade física de adolescentes colombianos e identificaram maiores pontuações em quase todas as dimensões de QVRS nos participantes provenientes de estratos socioeconômicos mais elevados. Isto se justifica devido a um maior acesso às melhores oportunidades para ocupar o tempo livre e realizar atividades físicas, refletindo uma percepção mais positiva da qualidade de vida.

Quanto à análise das relações entre as dimensões de qualidade de vida e o tempo de diagnóstico, observou-se correlação positiva e significativa na dimensão Autonomia. Tal resultado sugere que na medida em que o adolescente se adapta à sua nova condição, sua percepção acerca de sua autonomia e círculo de amizades é mais positiva (Salamon et al., 2010). Ao incorporar os seus regimes de saúde em seu estilo de vida, os adolescentes com DM1 percebem o autocuidado como uma parte natural de suas vidas (Karlsson, Arman, \& Wickblad, 2008).

Quando a adolescência é vivenciada juntamente com a experiência do diagnóstico e do tratamento de uma doença crônica, a intensidade das emoções pode ser potencializada. $\mathrm{O}$ adolescente pode se sentir inseguro ou incapaz de lidar com tantas informações e responsabilidades ao mesmo tempo. As restrições, em função do controle da doença, podem provocar alterações no cotidiano e afetar as relações interpessoais, a autoimagem e a autoestima. Em contrapartida, todas estas mudanças podem estimular a capacidade de adaptação, favorecendo o desenvolvimento de estratégias de enfrentamento.

O presente estudo demonstrou que a interferência da DM1 na QVRS de adolescentes afeta principalmente a percepção da dimensão saúde e atividade física, no entanto, os resultados também indicaram que, de modo geral, a QVRS foi avaliada de forma muito positiva. Contudo, cabe ressaltar que estes resultados devem ser considerados com cautela, pois como limitação do estudo, cita-se o número de participantes que não possibilitou análises estatísticas paramétricas com nível de erro amostral menor do que o de $10 \%$, o que pode ter diminuído o número de diferenças ou relações significativas.

A investigação da qualidade de vida relacionada à saúde do adolescente com doenças crônicas, como a diabetes mellitus, pode auxiliar na identificação de fatores de risco potenciais para o desenvolvimento biopsicossocial, bem como auxiliar no planejamento e na avaliação de intervenções em saúde pública que considerem a autopercepção e as necessidades dos adolescentes na sua relação com a família, amigos, escola e comunidade. 


\section{REFERÊNCIAS}

Associação Brasileira de Empresas de Pesquisa. (2016). Critério de Classificação Econômica, 2016. Recuperado de http://www. abep.org/criterio-brasil

Barros, L. (2003). Psicologia pediátrica: Perspectiva desenvolvimentista ( $2^{\mathrm{a}}$ ed.). Lisboa, Portugal: CLIMEPSI.

Bockhorst, C. L., Sumter, S. R., \& Westenberg, P. M. (2010). Social support from parents, friends, classmates, and teachers in children and adolescents aged 9 to 18 years: Who is perceived as most supportive? Social Development, 19(2), 417-426.

Bradley, R. H., \& Corwyn, R. F. (2002). Socioeconomic status and child development. Annual Review of Psychology, 53, 371-399.

Eiser, C., \& Morse, R. (2001). Quality-of-life measures in chronic diseases of childhood. Health Technology Assessment, 5(4), $1-157$.

Elamin, A., Hussein, O., \& Tuvemo, T. (2006). Growth, puberty, and final height in children with type 1 diabetes. Journal of Diabetes and its Complications, 20(4), 252- 256.

Gaspar, T., \& Matos, M. G. (Eds.). (2008). Qualidade de vida em crianças e adolescentes: Versão portuguesa dos instrumentos KIDSCREEN-52. Estrada da Costa, Portugal: Aventura Social \& Saúde.

Gaspar, T., Matos, M., Ribeiro, J., Leal, I., \& Ferreira, A. (2009). Health-related quality of life in children and adolescents and associated factors. Journal of Cognitive and Behavioral Psychotherapies, 9(1), 33-48.

González, E. J. O., Vélez, E. F. A., Rodríguez, C. M. V., Contreras, A. M. M., Restrepo, A. E., Berrió, G. B., ... Ochoa, G. M. A. (2014). Calidad de vida relacionada con la salud en un grupo de adolescentes de Medellín (Colombia): Asociación con aspectos sociodemográficos, exceso de peso u obesidad y actividad física. Revista Colombiana de Psicología, 23(2), 255-267.

Guedes, D., \& Guedes, J. E. R. P. (2011). Tradução, adaptação transcultural e propriedades psicométricas do KIDSCREEN-52 para a população brasileira. Revista Paulista Pediátrica, 29(3), 364-371.

Guedes, D. P., Astudillo, H. A.V., Morales, J. M. M., del Campo Vecino, J., \& Pires Júnior, R. (2014). Calidad de vida relacionada con la salud de adolescentes latinoamericanos. Revista Panamericana de Salud Pública, 35(1), 46-52.

International Diabetes Federation. (2017). Diabetes atlas (8 ed.). Brussels, Belgium: International Diabetes Federation. Recuperado de: https://diabetesatlas.org/IDF_Diabetes_ Atlas 8e interactive ES/

Kakleas, K., Kandyla, B., Karayianni, C., \& Karavanaki, K. (2009). Psychosocial problems in adolescents with type 1 diabetes mellitus. Diabetes \& Metabolism, 35(5), 339-350.

Karlsson, A., Arman, M., \& Wikblad, K. (2008). Teenagers with type 1 diabetes - A phenomenological study of the transition towards autonomy in self-management. International Journal of Nursing Studies, 45(4), 562-570.

Mackey, E. R., Hilliard, M. E., Berger, S. S., Streisand, R., Chen, R., \& Holmes, C. (2011). Individual and family strengths: An examination of the relation to disease management and metabolic control in youth with type 1 diabetes. Families, Systems, \& Health, 29(4), 314-326.

Matos, M. G. de, Gaspar, T., Simões, C., Borges, A., Camacho, I., Tomé, G., ... Equipa Aventura Social. (2012). KIDSCREEN: Qualidade de vida e saúde em crianças e adolescentes Relatório do Estudo KIDSCREEN 2010 e 2006. Lisboa, Portugal: Editora FMH/Universidade Técnica de Lisboa.
Michel, G., Bisegger, C., Fuhr, D. C., Abel, T., \& The KIDSCREEN Group. (2009). Age and gender differences in health-related quality of life of children and adolescents in europe: A multilevel analysis. Quality of Life Research, 18(9), 1147-1157.

Naar-King, S., Idalski, A., Ellis, D., Frey, M., Templin, T., Cunningham, P. B., ... Cakan, N. (2006). Gender differences in adherence and metabolic control in urban youth with poorly controlled type 1 diabetes: The mediating role of mental health symptoms. Journal of Pediatric Psychology, 31(8), 793-802.

Oliveira, J. E. P., \& Vencio, S. (Orgs.). (2016). Diretrizes da Sociedade Brasileira de Diabetes (2015-2016). São Paulo: A. C. Farmacêutica. Recuperado de http://www.diabetes.org.br/ sbdonline/images/docs/DIRETRIZES-SBD-2015-2016.pdf

Ottova, V., Erhart, M., Rajmil, L., Dettenborn-Betz, L., \& RavensSieberer, U. (2012). Overweight and its impact on the healthrelated quality of life in children and adolescents: Results from the european KIDSCREEN survey. Quality of Life Research, 21(1), 59-69.

Paredes, T., Simões, M. R., Canavarro, M. C., Serra, A. S. V., Pereira, M., Quartilho, M. J., ... Carona, C. (2008). Impacto da doença crónica na qualidade de vida: Comparação entre indivíduos da população geral e doentes com tumor do aparelho locomotor. Psicologia, Saúde \& Doenças, 9(1), 73-87.

Patton, G. C., \& Viner, R. (2007). Pubertal transitions in health. Lancet, 369(31), 1130-1139.

Perez, L. C. (2013). Adolescentes com diabete melito tipo 1: Resiliência, qualidade de vida, e suporte social (Dissertação de Mestrado). Programa de Pós-Graduação em Psicologia, Universidade Federal do Rio Grande do Sul, Porto Alegre, Rio Grande do Sul.

Peters, C. D., Storch, E. A., Geffken, G. R., Heidgerken, A. D., \& Silverstein, J. H. (2008). Victimization of youth with type-1 diabetes by teachers: Relations with adherence and metabolic control. Journal of Child Health Care, 12(3), 209-220.

Rajmil, L., Alonso, J., Berra, S., Ravens-Sieberer, U., Gosch, A., Simeoni, M. C., ... Auquier, P. (2006). Use of a children questionnaire of health-related quality of life (KIDSCREEN) as a measure of needs for health care services. Journal of Adolescent Health, 38(5), 511-518.

Salamon K. S., Hains, A. A., Fleischman, K. M., Davies, W. H., \& Kichler, J. (2010). Improving adherence in social situations for adolescents with type 1 diabetes mellitus (T1DM): A pilot study. Primary Care Diabetes, 4(1), 47-55.

Seiffge-Krenke, I., \& Stemmler, M. (2003). Coping with everyday stress and links to medical and psychosocial adaptation in diabetic adolescents. Journal of Adolescent Health, 33(3), 180-188.

Siegel, S., \& Castellan, N. J. Junior. (2006). Estatística não paramétrica para ciências do comportamento ( $2^{\mathrm{a}}$ ed.). Porto Alegre: Artmed.

Smolak, L. (2004). Body image in children and adolescents: Where do we go from here? Body Image, 1(1), 15-28.

Statsoft. (2011). Statistica for Windows [Computer program manual]. Versão 10.0. Tulsa: StatSoft. Recuperado de: http:// www.statsoft.com

World Health Organization. (2016). Global report on diabetes. Geneva: WHO. Recuperado de: http://apps.who.int/iris/bitstr eam/10665/204871/1/9789241565257_eng.pdf 